\title{
Pre induction cervical ripening in an outpatient setting
}

\author{
(Extract from the Dr. Nalin Rodrigo Memorial Oration - June 2018) \\ M Goonewardene ${ }^{\mathrm{a}}$
}

\section{Introduction}

Spontaneous onset of labour (SOL) is preceded by significant structural changes in the cervix which makes it softer and 'ripe', and enables it to stretch and dilate during labour. This remodeling or 'ripening' of the cervix involves increased turnover of its collagen, and the depletion of its mature, cross-linked collagen which is replaced by less mature, loosely packed collagen, resulting in a progressive reduction in its stiffness. These changes are influenced by numerous factors including changes in the composition of the extra cellular matrix, increased vascularization and hydration of the tissues, changes in barrier properties of the cervical epithelium and an influx of tissue monocytes. During cervical ripening there is also an alteration in local steroid concentrations in the cervical microenvironment with increased estradiol synthesis and a net loss of local progesterone ${ }^{1}$. Nitric oxide (NO), interacting with numerous substances including cytokines and free radicals, is thought to play an important role in initiating this process of cervical ripening ${ }^{2,3}$. This structural remodeling or ripening of the cervix is probably followed by further interlinked, harmonized changes in the levels of estrogen, progesterone, prostaglandins, corticotrophin releasing hormone and cortisol as well as their receptors, and all these are thought to lead to the initiation of labour ${ }^{3}$.
Spontaneous ripening of the cervix occurs gradually over several days or even weeks and there is no clear demarcation between ripening of the cervix and SOL because the former merges in to the latter in one continuous process. Therefore in clinical practice, most interventions used to ripen the cervix prior to induction of labour (IOL) will lead to IOL with stimulation of the uterine muscle contractions, if used in increasing doses and frequency and for longer durations. An ideal cervical ripening agent however, should result in cervical ripening only, without stimulating uterine contractions. Prior to the inclusion of prostaglandins as a method of IOL, amniotomy combined with an intravenous infusion of oxytocin was the commonest method of IOL used worldwide for several decades and delivery was expected within a maximum of 24 hours, as longer induction to delivery intervals with ruptured membranes are associated with increasing risks of chorio amnionitis and adverse outcomes. IOL with an "unfavourable cervix" resulted in an increased risk of caesarean delivery (CD) after 24 hours, which was carried out for a "failed induction". Therefore, for several decades various strategies have been adopted to ripen the cervix and make it "favourable" prior to IOL with amniotomy. Favourability for IOL is commonly assessed by the Bishop Score and its modifications ${ }^{4,5}$.

Sri Lanka Journal of Obstetrics and Gynaecology

2018; 40: 63-66

DOI: http://doi.org/10.4038/sljog.v40i3.7857

\footnotetext{
${ }^{\text {a }}$ Professor, Department of Obstetrics and Gynaecology, International Medical University, Clinical Campus Seremban, Malaysia.
}

Correspondence: MG, e-mail: <indramalikrodrigo@imu.edu.my>

http://orcid.org/0000-0002-7899-7117

Competing interest: The author reports no conflict of interest

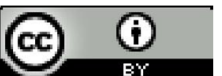

This is an open-access article distributed under the terms of the Creative Commons Attribution 4.0 International License, which permits unrestricted use, distribution and reproduction in any medium provided the original author and source are credited. 
During the last decade there has been an increasing interest in the possibility of cervical ripening and induction of labour in an outpatient setting, focusing on patent centered decision making and care. Most women who have received outpatient cervical ripening have been very positive and satisfied with their expe-rience, especially those who were able to self administer vaginallly the NO donor iso sorbide mononitrate (ISMN) and thus have had greater involvement and control over the process in a comfortable and supportive home environment. However, women who reside a significant distance away from their hospital may feel 'safer' in hospital and their preferences may vary according to the services available to them as well as their socio-demographic backgrounds ${ }^{6-9}$. Vaginally administered NO donors probably fit the criteria for ideal cervical ripening agents because they result in cervical ripening only, without stimulating uterine contractions, and ISMN is a cheap and freely NO donor which can be administered vaginally.

\section{Research on the vaginally administered nitric oxide donor iso sorbide mononitrate for pre- induction cervical ripening, carried out in a Teaching Hospital in Sri Lanka}

Four double-blind randomised controlled trials (RCT) have been carried out. Ethical approval for these studies was obtained from the Ethical Review Committee of the Faculty of Medicine, University of Ruhuna. Administrative approval was obtained from the Directors of the THMG during the periods of study. The RCTs were registered in the Sri Lanka Clinical Trial Register from November 2006 (the date the SLCTR was established). Informed written consent was obtained from all the participants in the trials. The RCTs listed are summarized below

1) The first RCT was in 2003/2004.

Objective: To determine whether sustained release ISMN-SR $60 \mathrm{mg}$ administered vaginally was effective for pre induction cervical ripening at 41 weeks' gestation.

Method: Using computer generated random numbers and concealment of the allocation in sequentially numbered sealed opaque envelopes, 156 consecutive women with uncomplicated singleton pregnancies at 41 weeks' gestation with a modified Bishop Score (MBS) <5 were allocated by stratified (primip/multip) block randomization in a $1: 1$ ratio to receive either ISMN-SR $60 \mathrm{mg}(\mathrm{n}=78)$ or vitamin C $100 \mathrm{mg}$ $(n=78)$ vaginally. The patients were blind to the type of intervention. The MBS before and after the intervention was assessed in all participants by one investigator who was adequately trained and skilled in this procedure, and blind to the intervention.

Results: At the commencement of the study there were no significant differences between the mean age, parity or MBS of the two groups. There were no dropouts from the study. In the ISMN$\mathrm{SR}$ group, there was a marked increase in the proportion establishing SOL (28\% vs $7.5 \%$, $\mathrm{p}<0.01)$ and becoming favourable for IOL $(40 \%$ vs $9 \% \mathrm{p}<0.001)$, two days after therapy. In the ISMN-SR group, there was also a significantly higher increase in the mean MBS $(3.8,95 \%$ CI 2.3-5.3 vs $1.3,95 \%$ CI $0.3-2.2, \mathrm{p}<0.01)$ and a marked decrease in the proportion of subjects requiring further ripening of the cervix with a supra cervical Foley catheter. (32\% vs $79 \%$, $\mathrm{p}<0.001)$. The CD rates were similar in both groups. Although assessment of $\mathrm{MBS}$ and its changes are subjective, SOL is an objective outcome which is not liable to patient or investigator bias.

Conclusion: ISMN-SR $60 \mathrm{mg}$ administered vaginally is effective for pre induction cervical ripening for post dated pregnancies at 41 weeks' gestation $^{10}$.

2) The second RCT was in 2007/2008.

Objective: To evaluate the effects on the mother and the fetus when ISMN $40 \mathrm{mg}$ or ISMN-SR 60 $\mathrm{mg}$ was administered vaginally for pre induction cervical ripening

Method: It was similar to the first study. However this study involved 156 women between 40 weeks +5 days and 41 weeks' gestation who were allocated to receive either ISMN 40 $\mathrm{mg}(\mathrm{n}=52)$ or ISMN-SR $60 \mathrm{mg}(\mathrm{n}=52)$ or the placebo vitamin C $100 \mathrm{mg}(\mathrm{n}=52)$ vaginally.

Results: At the commencement of the study the parity and mean age, MBS, pulse rate (PR), systolic and diastolic blood pressure (SBP and DBP), umbilical artery resistance index (RI) and pulsatility index (PI) were not significantly different among the three treatment groups. There were no dropouts from the study. There was a significant increase $(\mathrm{p}<0.001)$ of mean MBS by 1.3 (95\% CI $0.8-1.7$ ) in primips and by 1.7 (95\% CI 1.3-2.0) in multips at 6 hours and by 2.4 (95\% CI 1.9-2.7) in primips and by $2.3(95 \%$ CI 2.0-2.6) in multips at 48 hours. Greater proportions of primips ( $42 \%$ with ISMN-SR 
$60 \mathrm{mg}$ and $31 \%$ with ISMN $40 \mathrm{mg}, \mathrm{p}<0.05)$ were favourable for IOL after 48 hours in comparison with the controls $(7.6 \%)$. Greater proportions of multips (46\% with ISMN-SR $60 \mathrm{mg}$ and $40 \%$ with ISMN $40 \mathrm{mg}, \mathrm{p}<0.05)$ were favourable for IOL after 48 hours in comparison with the controls $(16 \%)$. A mean increase of PR by $6.7-$ 10.2 bpm (95\% CI 5.0-12.5, p<0.001) in both ISMN groups at 180 minutes persisted up to 360 minutes. A mean reduction of SBP by 7.3-10 $\mathrm{mmHg}(95 \% \quad \mathrm{CI}=8.0-11.5, \mathrm{p}<0.001)$ in both ISMN groups at 180 minutes persisted up to 360 minutes. No significant changes were seen in DBP, RI or PI. Frequency of maternal side effects (mainly headache) were higher in ISMN groups.

Conclusions: Although vaginal ISMN $40 \mathrm{mg}$ led to significantly greater cervical ripening after six hours, vaginal ISMN-SR $60 \mathrm{mg}$ was more effective than ISMN $40 \mathrm{mg}$ at 48 hours. Vaginal ISMN did not affect placental blood flow but caused headaches and clinically insignificant maternal haemodynamic changes ${ }^{11}$.

3) The third RCT was in 2011/2012.

Objective: To compare the effectiveness of two different treatment regimens of vaginal ISMNSR for pre-induction cervical ripening, and to assess its acceptability as an outpatient procedure.

Method: It was similar to the earlier studies. However this study involved 166 women who were allocated to receive vaginal ISMN-SR 60 $\mathrm{mg}$ at 40 weeks +3 and 5 days gestation as inpatients (Group A, n=74), or at 40 weeks $+3,4$ and 5 days gestation as inpatients (Group B, $\mathrm{n}=67$ ), or at 40 weeks $+3,4$ and 5 days gestation as outpatients (Group C, $\mathrm{n}=25$ ).

Results: Of those randomized to the out patient arm, only 25 received the therapy. This is because there were floods in the Southern Province of SL which prevented us from discharging patients and sending them home in the out patient therapy arm. At the commencement of the study there were no significant differences between the mean age, parity or MBS among the three groups. Mean increase of MBS was markedly greater $(3.0,95 \%$ CI $2.7-3.3$ vs. 1.6 , $95 \%$ CI 1.2-1.9, $\mathrm{p}<0.001)$ and the proportions with MBS > 6 were markedly greater in Group B compared to Group A (83\% vs 32\%, Relative Risk 2.57 95\% CI 1.76-3.77, p<0.001). Selfadministration of ISMN was noted in $60 \%$, and
$80 \%$ were satisfied with it and happy to recommend it to another patient. Mean visual analogue score for nausea and vomiting was least in Group $\mathrm{C}(\mathrm{p}=0.002)$.

Conclusions: Three days of ISMN-SR $60 \mathrm{mg}$ vaginally was better than the two day regimen for pre induction cervical ripening, and outpatient therapy was acceptable to patients ${ }^{12}$.

4) The fourth RCT was in 2014/2015.

Objective: To determine the effectiveness and acceptability of ISMN self-administered vaginally at home, in causing cervical ripening in uncomplicated singleton pregnancies at 39 weeks' gestation.

Method: It was similar to the earlier studies. However this study involved 144 women who were allocated to self-administer vaginally at home every other day, five doses of $60 \mathrm{mg}$ of ISMN-SR ( $\mathrm{n}=72$, cases), or Pyridoxine $10 \mathrm{mgs}$ ( $n=72$, controls), from 39 weeks' gestation to 40 weeks +2 days' gestation.

Results: At the commencement of the study there were no significant differences between the mean age, parity or MBS of the two groups There were no dropouts from the study. By 40 weeks +2 days gestation,the mean MBS, the mean change of MBS and the proportions with SOL, were not significantly different between the two groups. A vast majority were satisfied with outpatient therapy ( $80 \%$ in cases vs. $76 \%$ in controls), were happy to use it in a subsequent pregnancy $(89 \%$ in cases vs. $86 \%$ in controls), and would recommend it to a friend. (93\% in cases vs. $90 \%$ in controls).

Conclusions: Outpatient vaginal ISMN therapy from 39 weeks' gestation was not effective in causing significant cervical ripening but it was acceptable to women ${ }^{13}$.

Therefore in summary we concluded that:

1) Sustained release ISMN $60 \mathrm{mg}$ administered vaginally is effective for pre induction cervical ripening for post dated pregnancies at 41 weeks' gestation, and a three day regimen is better than a two day regimen ${ }^{10,12}$.

2) Although vaginal ISMN $40 \mathrm{mg}$ results in significantly greater cervical ripening after six hours, vaginal ISMN-SR $60 \mathrm{mg}$ is more effective than ISMN $40 \mathrm{mg}$ at 48 hours $^{11}$.

3) Vaginal ISMN could be considered safe as it did not affect placental blood flow. It can cause moderate headaches and clinically 
insignificant haemodynamic changes in the mother ${ }^{11}$.

4) Outpatient ISMN therapy is acceptable to patients although it is not effective from 39

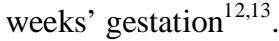

\section{Conclusions}

As outpatient vaginal ISMN therapy has been found to be feasible and acceptable to women, it should be considered as an option for pre induction cervical ripening for women with post dated pregnancies in Sri Lanka. As stated in the most recent Cochrane review on this subject, vaginally administered NO donors such as ISMN are effective and can be safely used for outpatient cervical ripening after 40 weeks gestation in low risk women ${ }^{14}$. Further research is needed to identify the optimum formulation, dose, frequency and duration of therapy, as well as its role as an adjunct intervention to improve the outcome when it is combined with other agents for IOL in post dated pregnancies. However, they may be ineffective prior to 40 weeks gestation ${ }^{13}$. A more robust set of outcome measures, which enable the cervical ripening phase of the induction process to be effectively evaluated, must also be developed ${ }^{14}$.

\section{References}

1. Timmons B, Akins M, Mahendroo M. Cervical remodeling during pregnancy and parturition. Trends Endocrinol Metab. 2010; 21(6): 353-61.

2. Chwalisz K, Garfield RE. Nitric oxide as the final mediator of cervical ripening. Human Reproduction 1998;13: 245-52.

3. Kamel RM. The onset of human parturition. Arch Gynecol Obstet 2010;281: 975. doi:10.1007/s00404-010-1365-9. [Accessed 02.02.2018].

4. Bishop EH. Pelvic scoring for elective induction. Obstet Gynecol 1964; 24: 266-8.

5. Biswas A, Arulkumaran S. Induction of labour. In: Arulkumaran S, Ratnam SS, Bhasker Rao K (eds). The Management of Labour, Vol. 14. Madras: Orient Longman Ltd, 1996; 213-27.

6. Moore JE. Moving Towards Patient Centered Care: Women's Decisions, Perceptions, and Experience of the Induction of Labor Process. A dissertation submitted in partial fulfillment of the requirements for the degree of Doctor of Philosophy (Nursing).

The University of Michigan 2012.

[Accessed 02.02.2018].

7. Oster C, Adelson PL, Wilkinson C, Turnbull D. Inpatient versus outpatient cervical priming for induction of labour: Therapeutic landscapes and women's preferences. Health \& Place 2011; 17: 379-85.

8. Reid M, Lorimer K, Norman JE, Bollapragada SS, Norrie J. The home as an appropriate setting for women undertaking cervical ripening before the induction of labour. Midwifery 2011; 27: 30-5.

9. Howard K, Gerard K, Adelson P, Bryce R, Wilkinson C, Turnbull D. Women's preferences for inpatient and outpatient priming for labour induction: a discrete choice experiment. BMC Health Services Research 2014, 14: 330-41.

10. Rameez MFM, Goonewardene IMR. Nitric oxide donor iso-sorbide mono nitrate for pre induction cervical ripening at 41 weeks' gestation: a randomized controlled trial. J Obstet Gynaecol Res 2007; 33 (4): 452-7.

11. Widanagamage RS, Goonewardene IMR. The efficacy of two different doses of vaginal isosorbide mono nitrate in pre induction cervical ripening: a double blind randomized controlled trial Ceylon Med J 2011; 56(3): 91-100.

12. Ziard MH, Goonewardene IMR. Efficacy of two different treatment regimens of vaginal Nitric Oxide donor (Iso-sorbide mononitrate) use $\mathrm{d}$ for pre induction cervical ripening.

Abstracts of the $45^{\text {th }}$ Scientific Session of the Sri Lanka College of Obstetrics and Gynaecology, Sri Lanka J Obst and Gynaecol 2012; 34(1): OP13,pg 81.

13. Attanayake K, Goonewardene M, Outpatient cervical ripening with the nitric oxide donor isosorbidemononitrate vaginally, in uncomplicated singleton pregnancies at 39 weeks gestation: a double blind randomized controlled trial. Ceylon Med J 2016; 61: 142-8.

14. Ghosh A, Lattey KR, Kelly AJ. Nitric oxide donors for cervical ripening and induction of labour. Cochrane Database of Syst Rev 2016, Issue 12. Art. No.: CD006901. doi: 10.1002/14651858.CD006901.pub3. [Accessed 02.02.2018]. 